\title{
Dimethylation of Histone 3 Lysine 9 is sensitive to the epileptic activity, and affects the transcriptional regulation of the potassium channel Kcnj10 gene in epileptic rats
}

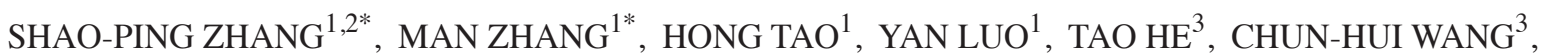 \\ XIAO-CHENG LI ${ }^{3}$, LING CHEN ${ }^{1,3}$, LIN-NA ZHANG ${ }^{1}$, TAO SUN ${ }^{2}$ and QI-KUAN HU ${ }^{1-3}$
}

${ }^{1}$ Department of Physiology; ${ }^{2}$ Ningxia Key Laboratory of Cerebrocranial Diseases, Incubation Base of National Key Laboratory, Ningxia Medical University; ${ }^{3}$ General Hospital of Ningxia Medical University, Yinchuan, Ningxia 750004, P.R. China

Received February 18, 2017; Accepted September 13, 2017

DOI: $10.3892 / \mathrm{mmr} .2017 .7942$

\begin{abstract}
Potassium channels can be affected by epileptic seizures and serve a crucial role in the pathophysiology of epilepsy. Dimethylation of histone 3 lysine 9 (H3K9me2) and its enzyme euchromatic histone-lysine $\mathrm{N}$-methyltransferase 2 (G9a) are the major epigenetic modulators and are associated with gene silencing. Insight into whether H3K9me2 and G9a can respond to epileptic seizures and regulate expression of genes encoding potassium channels is the main purpose of the present study. A total of 16 subtypes of potassium channel genes in pilocarpine-modelled epileptic rats were screened by reverse transcription-quantitative polymerase chain reaction, and it was determined that the expression ATP-sensitive inward rectifier potassium channel 10 (Kcnj10) increased in hippocampus and insular cortex, while the expression of most of the other subtypes decreased. The total level of H3K9me2 decreased in the model group compared with the control. The Kcnj10 gene encoding the Kir4.1 channel was selected to analyse changes in $\mathrm{H} 3 \mathrm{~K} 9 \mathrm{me} 2$ in the promoter region by the chromatin immuno-precipitation method. Anti-H3K9me2 and anti-G9a antibodies were used to identify the modified DNAs. Five primers were designed across the promoter region of the Kcnj10 gene. In epileptic hippocampi, the relative abundance of $\mathrm{H} 3 \mathrm{~K} 9 \mathrm{me} 2$ and G9a in the promoter region of Kcnj10 decreased markedly. Removal of the H3K9me2 repressive mark resulted in decreased transcriptional inhibition of the Kcnj10 gene and therefore increased its expression. In the cultured C6 cells, specific inhibition of the enzymatic activity of
\end{abstract}

Correspondence to: Dr Qi-Kuan Hu, Department of Physiology, Ningxia Medical University, 1160 Shengli Street, Yinchuan, Ningxia 750004, P.R. China

E-mail: huqikuan@163.com

*Contributed equally

Key words: enzyme euchromatic histone-lysine N-methyltransferase 2, dimethylation of histone $\mathrm{H} 3$ at lysine 9, ATP-sensitive inward rectifier potassium channel 10, potassium channel subtypes, epilepsy
G9a by 2-(Hexahydro-4-methyl-1H-1,4-diazepin-1-yl)-6,7-dimethoxy-N-(1-(phenyl-methyl)-4-piperidinyl)-4-quinazolinamine tri-hydrochloride hydrate (bix01294) resulted in upregulation of the expression of Kir4.1 proteins. The present study demonstrated that $\mathrm{H} 3 \mathrm{~K} 9 \mathrm{me} 2$ and $\mathrm{G} 9 \mathrm{a}$ are sensitive to epileptic seizure activity during the acute phase of epilepsy and can affect the transcriptional regulation of the Kcnj10 channel.

\section{Introduction}

Epilepsies are disorders of neuronal excitability, characterized by spontaneous and recurrent seizures. Potassium ion channels serve an important role in the pathogenesis of epilepsy. They are the core targets for excitability regulation and are involved in potassium homeostasis. Potassium ion channels adjust the cell membrane resting potential and determine the amplitude and frequency of action potentials, and buffer the extracellular potassium ions. Over 80 subtypes of potassium ion channels have been identified to date on the surface of neuronal and glial cells (1). Several epileptic phenotypes have been associated with dysfunctions of potassium channels. Changes in the extracellular $\mathrm{K}^{+}$concentration represent one of the earliest and most thoroughly described examples of activity-induced changes to ion concentration gradients (2). Epileptic seizures are associated with the expression, distribution and density of various subtypes of potassium channels. A microarray of several potassium channel genes, Kcnmb4, Kcnd 2 and Kcnc1 were downregulated during the acute phase of epilepsy in the CA3 region of the hippocampus (3). Although the aforementioned studies revealed that potassium channels can be regulated during epilepsy, the exact mechanisms underlying these observations remain to be elucidated.

An increasing amount of evidence suggests that epigenetic mechanisms are involved in the regulation of potassium channels in various neural pathogeneses $(4,5)$. The epigenetic mechanism involved in the potassium homeostasis and the dynamics of epilepsy remain to be elucidated. Downregulated Kir4.1 protein expression is observed in several human central nervous system (CNS) pathologies, including CNS ischemic injury, spinal cord injury, epilepsy, amyotrophic lateral sclerosis and Alzheimer's disease (reviewed in 6). Studies on Kir4.1 
knockout mice indicated that DNA methylation is a key regulator of Kir4.1 transcription during CNS development (7). DNA hydroxymethylation also regulates the differential expression of different subtypes of potassium channels in drug-addiction behaviours (8). Nerve injury increases the dimethylation of histone $\mathrm{H} 3$ at lysine 9 (H3K9me2) at potassium voltage-gated channel subfamily A member 4, subfamily D member 2(Kcnd2), subfamily KQT member 2 and calcium-activated potassium channel subunit alpha-1 promoters, but does not affect the levels of DNA methylation in the dorsal root ganglion (9). Histone deacetylase inhibitors modulate ATP-sensitive potassium channel subunit transcription at the SUR2 promoter in HL-1 cardiomyocytes (10). In a genome-wide DNA methylation analysis, KCNN4 (KCa 3.1 channel) promoter was identified to be hypo-methylated in an aggressive non-small-cell lung carcinoma cell line and in patient samples (11).

One of the major epigenetic modulations is histone $\mathrm{H} 3$ at lysine 9 (H3K9) methylation. It includes mono-, di- and tri-methylations, which are catalysed by several histone methyltransferases, such as histone-lysine N-methyltransferase EHMT2 (G9a) and histone-lysine N-methyltransferase SUV39H1. H3K9me2 is the main repressive mechanism of the transcriptional regulation of gene expression, and is commonly associated with gene silencing. G9a mediates H3K 9 mono- and di-methylation (H3K9me1/2) and targets euchromatic loci (12). In the present study, it was identified that the expression of $\mathrm{H} 3 \mathrm{~K} 9 \mathrm{me} 2$ decreased markedly during the epileptic process, especially in the acute phase of epileptic seizure. The downregulation of $\mathrm{H} 3 \mathrm{~K} 9 \mathrm{me} 2$ was associated with an upregulated expression of several subtypes of potassium channels, including Kir4.1. These observations suggest that $\mathrm{H} 3 \mathrm{~K} 9 \mathrm{me} 2$ serves a role in the regulation of epileptic seizures and the transcriptional regulation of potassium channel genes, which was investigated further in the present study.

\section{Materials and methods}

Animals and epilepsy model. All experimental procedures in the present study were approved by the Animal Ethics and Use Committees of Ningxia Medical University (Yinchuan, China).

Healthy male Sprague Dawley rats (weight, 280-300 g; aged 8-12 weeks) obtained from the Animal Centre of Ningxia Medical University were reared at 6 per cage at $24.0 \pm 0.2^{\circ} \mathrm{C}$ in a humidified atmosphere $(54 \pm 5 \%)$. Rats were fed with a standard rodent animal feed, were specific-pathogen-free and kept under a natural circadian rhythm, with natural lighting and regular ventilation. Rats were randomly divided into healthy control and pilocarpine epilepsy groups. Totally 40 rats were used, with 25 in the epilepsy group and 15 in the control group. More specifically, 15 epileptic rats and 5 control rats for western blotting; 5 epileptic rats and 5 control rats for RT-qPCR and 5 epileptic rats and 5 control rats for ChIP assay (Table I). The epilepsy model was established using lithium chloride (LiCl)/Pilocarpine (Sigma-Aldrich; Merck KGaA, Darmstadt, Germany) as previously described (13). Rats were injected intraperitoneally with $\mathrm{LiCl}(127 \mathrm{mg} / \mathrm{kg})$ and then with pilocarpine $(30 \mathrm{mg} / \mathrm{kg}) 18$ to $24 \mathrm{~h}$ following the first injection. Rats with Racine IV-V grade epileptic seizures were administered chloral hydrate $(300 \mathrm{mg} / \mathrm{kg})$ injection following $1 \mathrm{~h}$ of seizure, and were subsequently used in further experiments. Pilocarpine was administered to each animal up to 4 times (Table I). Time intervals between the first pilocarpine injections to the first IV-V grade epileptic seizure were recorded as seizure latency and are presented in Table I. Rats that received pilocarpine more than 6 times were not included in the present study. Rats in the control group were administered a $0.9 \%$ saline injection for 2 treatments with about $1 \mathrm{~h}$ interval.

Brain tissue preparation and western blotting. Rat brains were dissected, and hippocampal and insular cortex tissues were isolated and processed immediately for protein or RNA extraction. Each assay included 5 rats. For western blotting, a Total Protein Extraction kit (Nanjing KeyGen Biotech. Co., Ltd., Nanjing, China) was used, and the Bicinchoninic Acid assay was used for protein quantification (Nanjing KeyGen Biotech. Co., Ltd.) according to the manufacturer's protocol. The following antibodies were used: Anti-EHMT2/G9A (dilution, 1:1,000; cat. no. ab40542), anti-Kenj10 (dilution, 1:1,000; cat. no. ab192404), anti-histone H3 (di methyl K9; dilution, 1:1,000, cat. no. ab1220; all from Abcam, Cambridge, MA, USA) and anti-GAPDH (dilution, 1:5,000; cat. no. bsm-0798m; BIOSS, Beijing, China). Peroxidase-conjugated goat anti-rabbit IgG (cat. no. bs-0295G) and goat anti-mouse IgG (cat. no. bs-0296G) were used as the secondary antibodies (both dilutions, 1:10,000; BIOSS). All antibodies were diluted with 5\% skimmed milk (BD Biosciences, Franklin Lakes, NJ, CA, USA).

$R T-q P C R$. Total RNA was purified from rat brain tissues with TRIzol reagent (Invitrogen; Thermo Fisher Scientific, Inc., Waltham, MA, USA) according to manufacturer's protocol. The RNA was reverse-transcribed to cDNA using a Reverse Transcription system (Pomega Corporation, Madison, WI, USA) according to the manufacturer's protocol: $70^{\circ} \mathrm{C}$ for $5 \mathrm{~min}, 25^{\circ} \mathrm{C}$ for $5 \mathrm{~min}, 42^{\circ} \mathrm{C}$ for $60 \mathrm{~min}$, and $70^{\circ} \mathrm{C}$ for $15 \mathrm{~min}$. A total of 16 primer pairs specific to different potassium channel subtypes were designed and tested by the melting curve analysis (Table II). The following thermo cycling conditions were used qPCR: $50^{\circ} \mathrm{C}$ for $2 \mathrm{~min}, 95^{\circ} \mathrm{C}$ for $10 \mathrm{~min}$ and 40 cycles of $95^{\circ} \mathrm{C}$ for $15 \mathrm{sec}$, followed by $60^{\circ} \mathrm{C}$ for $1 \mathrm{~min}$. GAPDH served as a housekeeping gene. All RT-qPCR experiments were run in triplicate. The $2^{-\Delta \Delta \mathrm{Cq}}$ method (14) was used to determine the relative fold change in mRNA expression. Samples were analysed using a Smart Spec Plus spectrophotometer (Bio-Rad Laboratories, Inc., Hercules, CA, USA).

ChIP assay. A total of 5 epileptic and 5 control rats were used for ChIP assay. Anti-histone H3 (di methyl K9) antibody (dilution, 1:1,000; cat. no. ab1220; Abcam) and anti-EHMT2/G9A (dilution, 1:1,000; cat. no. ab40542; Abcam) antibodies, and an EZ-ChIP kit (Haoran Biological Technology Co., Ltd., Shanghai, China) were used. DNA fragments were sonicated to 100-500 bp. RT-qPCR was performed as described in the previous section. A total of 5 primer pairs specific to the Kcnj10 promoter region were designed, and the sequences are presented in Table II. Total genome DNA was used as a template. All samples were tested in triplicate and the results from each sample were normalized relative to the input genomic DNA.

Cell culture and treatments. The C6 glioma cell line (Shanghai Jing Kang Biological Engineering. Co., Ltd., Shanghai, China; 

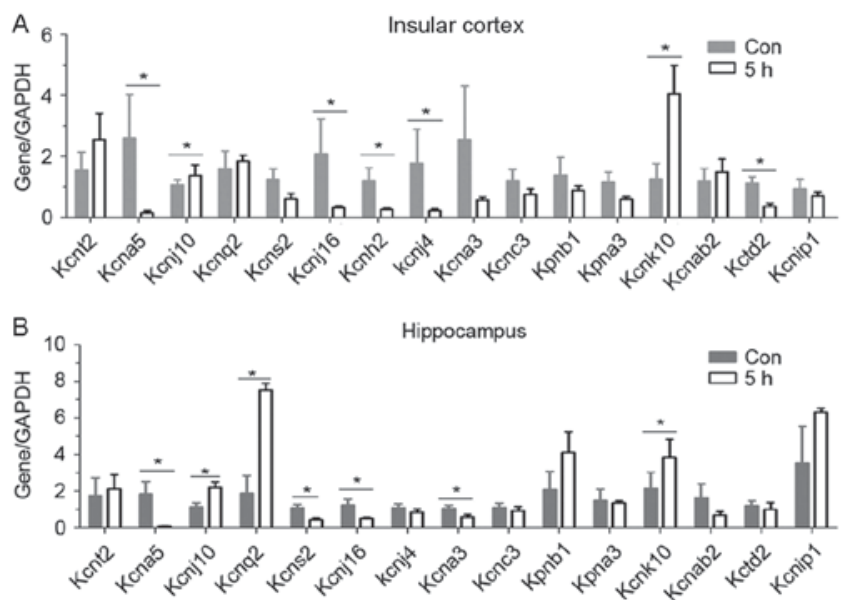

Figure 1. Screening of potassium channel subtypes by reverse transcription-quantitative polymerase chain reaction. Sixteen potassium subtypes were screened in (A and B) two brain regions of rats $5 \mathrm{~h}$ following epilepsy. Five rats were used in each group $(\mathrm{n}=5)$. Data are expressed as the mean \pm standard error. ${ }^{*} \mathrm{P}<0.05$ vs. the control group. Kcnt 2 , potassium channel subfamily $\mathrm{T}$ member 2 ; Kcna5, potassium voltage-gated channel subfamily A member 5; Kcnq2, potassium voltage-gated channel; Kcnq2, subfamily KQT member 2; Kcns2, subfamily S member 2; Kcnh2, subfamily $\mathrm{H}$ member 2; Kcna3, subfamily A member 3; Kcnj10, ATP-sensitive inward rectifier potassium channel 10; Kcnj10, ATP-sensitive inward rectifier potassium channel 10 ; Kcnj16, inward rectifier potassium channel 16; Kcnj4, inward rectifier potassium channel; Kpnb1, importin subunit beta-1; Kpna3, inward rectifier potassium channel alpha-4; Kcnk10 importin subunit potassium channel subfamily $\mathrm{K}$ member 10 ; Kcnab2, voltage-gated potassium channel subunit beta-2; Kctd2, BTB/POZ domain-containing protein KCTD2; Kcnip1, Kv channel-interacting protein 1; Con, control.

www.shjingk.com/) were cultured in high glucose Dulbecco's modified Eagle's Medium with $1 \%$ penicillin/streptomycin and $5 \%$ fetal bovine serum in a $5 \% \mathrm{CO}_{2}$ incubator at $37^{\circ} \mathrm{C}$ for $24 \mathrm{~h}$. A total of $1 \mathrm{mM} / \mathrm{ml}$ - (Hexahydro-4-methyl-1H-1,4-diazepin-1-yl) -6,7-di-methoxy-N-(1-(phenyl-methyl)-4-piperidinyl)-4-quinazolinamine tri-hydrochloride hydrate (Bix01294; Sigma-Aldrich; Merck KGaA,) was added to the medium at the final concentration of 1,2, 4 and $8 \mu \mathrm{M} / \mathrm{ml}$ for $5 \mathrm{~h}$. Proteins were extracted using the Cell Lysis \& Protein Extraction kit, containing inhibitors of phosphatases and protease inhibitors, from brain tissues or C6 cells (Cell Lysis \& Protein Extraction kit; Nanjing KeyGen Biotech. Co., Ltd., Nanjing, China). Briefly, tissues were pipetted in the lysis buffer on ice and rotated at $4^{\circ} \mathrm{C}$ for $30 \mathrm{~min}$ and then centrifuged for $40 \mathrm{~min}$ in $10,800 \mathrm{x} \mathrm{g}$ at $4^{\circ} \mathrm{C}$.

Statistical analysis. Statistical analyses were performed using SPSS (version 17.0; SPSS, Inc., Chicago, IL, USA). All data are presented as the mean \pm standard error. Differences between groups were analysed using Student's t-test or one-way analysis of variance with Dunnett's post hoc test. $\mathrm{P}<0.05$ was considered to indicate a statistically significant difference.

\section{Results}

Screening of the potassium channel subtypes by RT-qPCR. A total of 16 potassium channel subtypes including voltage-gated, inwardly rectifying, ATP sensitive, M type and $\mathrm{Ca}^{2+}$-stimulated, were screened by RT-qPCR. In the insular cortex, and in hippocampus, $5 \mathrm{~h}$ after seizure, Kcnj10 was upregulated (Fig. 1A and B). The other upregulated gene 
Table II. Primer sequences for RT-qPCR and ChIP.

RT-qPCR primer sequence $\left(5^{\prime} \rightarrow 3^{\prime}\right)$

\begin{tabular}{lll}
\cline { 2 - 3 } Gene & \multicolumn{1}{c}{ Forward } & \\
\hline KCNT2 & ACATCCCCAAAGCCCACA & TGGGAGCAGATTTTACGAGTTC \\
KCNA5 & GAGAATGACCAGGACCGACAC & TCATCAAGGAAGAGGAGAAGCC \\
KCNQ2 & GGATTCCGCCGTTTCTCA & ACCTCATTGGTGTCTCATTCTT \\
KCNJ10 & GGGGACGCCACTTTCACAA & GAGATCCTCTGGGGCTACGA \\
KCNS2 & TGCCCAAGTTAGCCAAGGT & AATCGTGGAGCACTTTGGC \\
KCNJ16 & GGGTCGGAAGTCACCTATGC & CATTGGGGCAGCCTTGG \\
KCNJ4 2 & GCCCCTCGGAATGGTTTG & TTCCATCAGATTCCCAACCC \\
KCNA3 & CGGAGATGACAGCGTGGTG & GCGGTCATCGCAGTGGTT \\
KCNC3 & TTACTGGGGCAAGCAAAGAAT & TCTTCTGCTTGGAGACACTACCC \\
KPNB1 & CAACGCCCAACTGCTACG & GACTCCCGTTCCCTCTTCG \\
KPNA3 & TGGATCATTGGCCTAGCTTCTA & AGCCCAAGTGGACAAGTCAGA \\
KCNK10 & TGGGACAGGACATCGCAGTT & AGCCACCAGGAAGTCAAAGTT \\
KCNAB2 & CCCTGCCACAAAATCACCA & ATCCCGCTCTTCGGTTTCT \\
KCTD2 & GCTCCAGCGTGATGTGCC & GAGAAGGGGTGGAGACGG \\
GAPDH & CGTTGTCCCGTATCCTTTCC & ACATCAACAAGGACGGATACATAAAC \\
\hline
\end{tabular}

Gene

ChIP primer sequence $\left(5^{\prime} \rightarrow 3^{\prime}\right)$

$1-\mathrm{KCNJ} 10$
$2-\mathrm{KCNJ} 10$
$3-\mathrm{KCNJ} 10$
$4-\mathrm{KCNJ} 10$
$5-\mathrm{KCNJ} 10$

CTCTGCCCTCTACACTCAT

\author{
ACATAGTGGATGGGAAGAT \\ ACAGGGAACAACGAAAAC \\ GACAAACCCTTATCTGATTC \\ TCCCTCGTGCTTTCATCG \\ GATAATCTCCCATTCCTAAC
}

RT-qPCR, real time-quantitative polymerase chain reaction; ChIP, chromatin immune-precipitation.

was Kcnk10. Many subtypes such as Kcna5, Kcnj16, Kcnh2, Kcnj4 andKctd2 in the insular (Fig. 1A) and Kcna5, Kcns2, Kcnj16, Kcna3 andKctd2 in the hippocampus (Fig. 1B), were downregulated. In the present study, the focus was on Kcnj10.

Upregulation of Kcnj10-encoded Kir4.1 protein in hippocampus. The expression of Kir4.1 was tested by western blotting with five epileptic and five control rats. Tissues were analysed 1, 5 and $24 \mathrm{~h}$ following an epileptic seizure. The protein expression levels of the Kir4.1 were upregulated at $24 \mathrm{~h}$ following an epileptic seizure in the insular cortex (Fig. 2A) and at $5 \mathrm{~h}$ following an epileptic seizure in the hippocampus (Fig. 2B). Compared with the control group, the expression of Kir4.1 proteins at $5 \mathrm{~h}$ following a seizure was increased in the hippocampus $(\mathrm{P}<0.05)$.

Downregulation of $H 3 \mathrm{~K} 9 \mathrm{me} 25 \mathrm{~h}$ following an epileptic seizure. In order to analyse the mechanism of epigenetic regulation of the potassium channel genes, the expression of H3K9me2 and its enzyme G9a was tested by a western blotting at three different time points: 1,5 and $24 \mathrm{~h}$. As presented in Fig. 3, H3K9me2 and G9a were significantly downregulated $5 \mathrm{~h}$ following a seizure, both in the hippocampus and insular cortex $(\mathrm{P}<0.05)$. The initial expression level was restored at 24 h (Fig. 3).

Decrease of H3K9me2 and G9a occupancy in the Kcnj10 promoter. The aforementioned results suggested an association between the $\mathrm{H} 3 \mathrm{~K} 9 \mathrm{me} 2$ modification and the transcriptional regulation of Kcnj10 gene. ChIP experiments were designed to analyse changes in levels of $\mathrm{H} 3 \mathrm{~K} 9 \mathrm{me} 2$ in the promoter region of the Kcnj10 gene. A total of 5 primer pairs specific to the Kcnj10 promoter region were designed to anneal across exon1 (Fig. 4A).

Relative levels of $\mathrm{H} 3 \mathrm{~K} 9 \mathrm{me} 2$ at primer annealing sites 1, 2,3 , and 4 decreased significantly $5 \mathrm{~h}$ following an epileptic seizure in the hippocampus (Fig. 4B).

In order to confirm whether the observed changes in methylation were catalysed by G9a, ChIP experiments using the anti-G9a antibody were performed to identify on-going methylation events. The abundance of G9a at primer annealing sites 2, 3 and 4 also decreased significantly $5 \mathrm{~h}$ following an epileptic seizure in the hippocampus (Fig. 4C). 
A
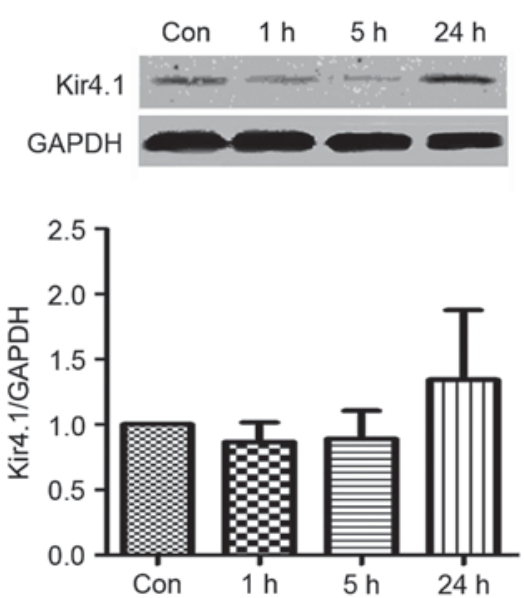

B
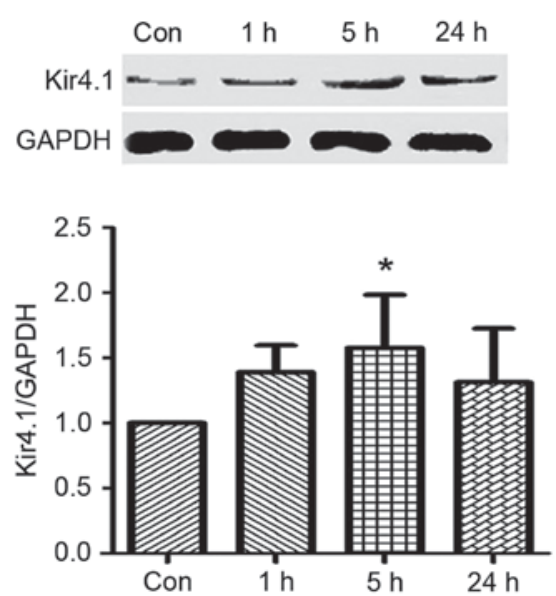

Figure 2. Western blotting of Kir4.1. The expression levels of Kir4.1 protein was significantly increased (A) $24 \mathrm{~h}$ following epileptic seizure in the insular cortex and (B) after $5 \mathrm{~h}$ in the hippocampus. Five rats were used in each group ( $\mathrm{n}=5$ ). Data are expressed as the mean \pm standard error. ${ }^{*} \mathrm{P}<0.05 \mathrm{vs}$. the control group. Con, control.
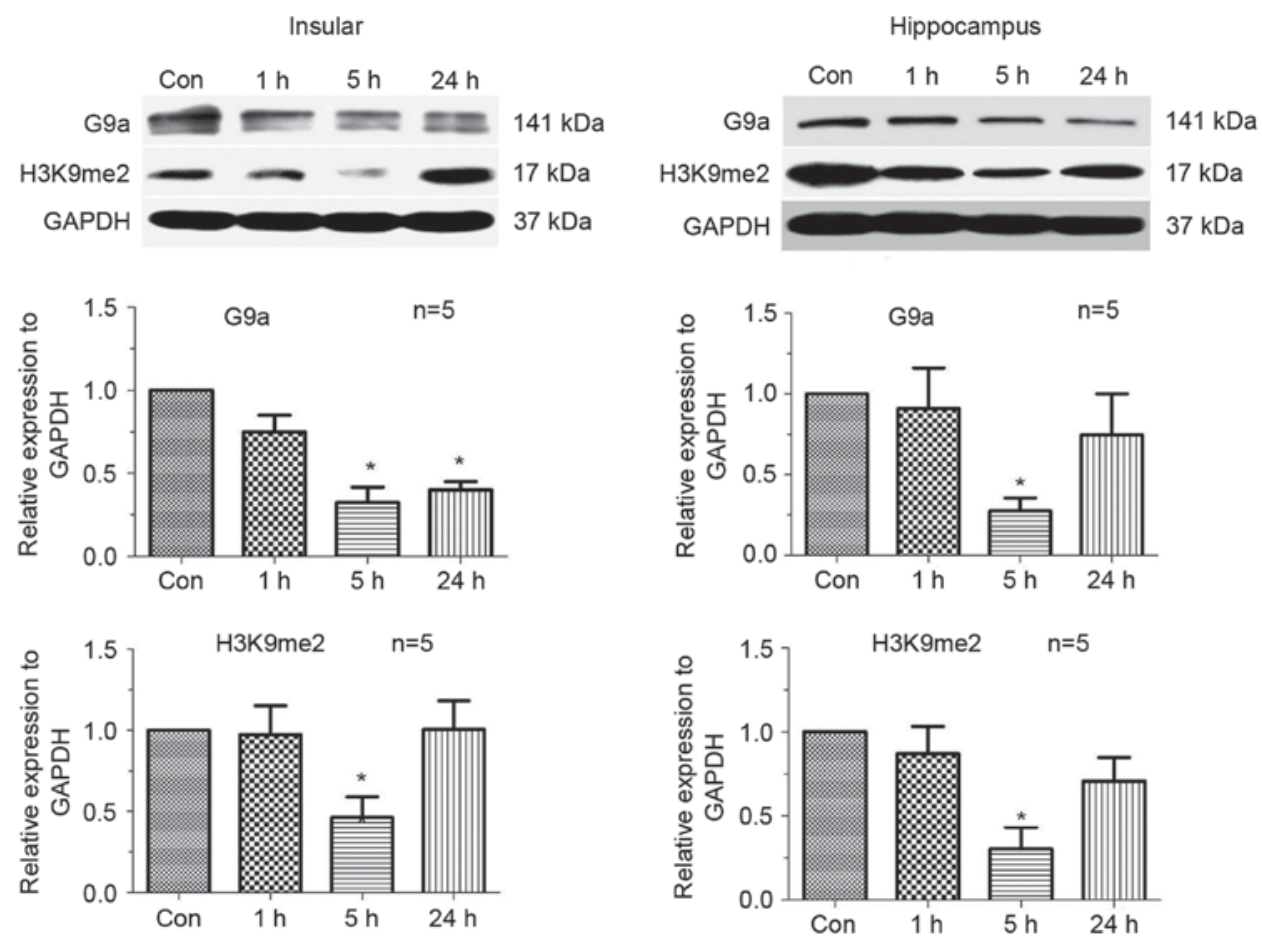

Figure 3. Western blotting of H3K9me2 and G9a. The expression levels of H3K9 and G9a were markedly decreased at 5 h post seizure, both in hippocampus and insular cortex, and then increased at the $24-\mathrm{h}$ time point. Five rats were used in each group $(\mathrm{n}=5)$. Data are expressed as the mean \pm standard error. $\mathrm{P}<0.05$ vs. the control group. H3K9me2 dimethylation of histone 3 lysine 9; G9a, euchromatic histone-lysine N-methyltransferase 2; Con, control.

Bix01294 inhibits G9a and H3K9me2 and increases the expression of Kir4.1 (Kcnj10). Bix01294, a specific G9a inhibitor, was used to verify Kcnj10 regulation by G9a. Following a 1 and $4 \mu \mathrm{mol} / 1$ treatments with bix01294 for $5 \mathrm{~h}$, the expression of G9a and H3K9me2 decreased in C6 cells, while the expression of Kir4.1 (Kcnj10) demonstrated an increase in a dose-dependent manner between 1 and $4 \mu \mathrm{mol} / 1$ doses (Fig. 5).

\section{Discussion}

Seizures can cause long-lasting changes in gene expression patterns. Epigenetic mechanisms may alter gene expression in response to seizure. Without causing mutations in gene sequences, epigenetic modifications, including histone methylation, may respond to changes inside the cell caused by seizure and alter gene transcription through chromatin adjustments. The present study investigated the association between epigenetic modification of the potassium channel expression and epileptic activities.

The present study demonstrated that $\mathrm{H} 3 \mathrm{~K} 9$ dimethylation and its enzyme G9a were sensitive to the seizure activity. Following a $1 \mathrm{~h}$-long seizure interrupted with $\mathrm{LiCl} /$ pilocarpine, H3K9me2 and G9a were downregulated. The downregulation lasted for $<24 \mathrm{~h}$, was lowest at $5 \mathrm{~h}$ following the seizure 
A
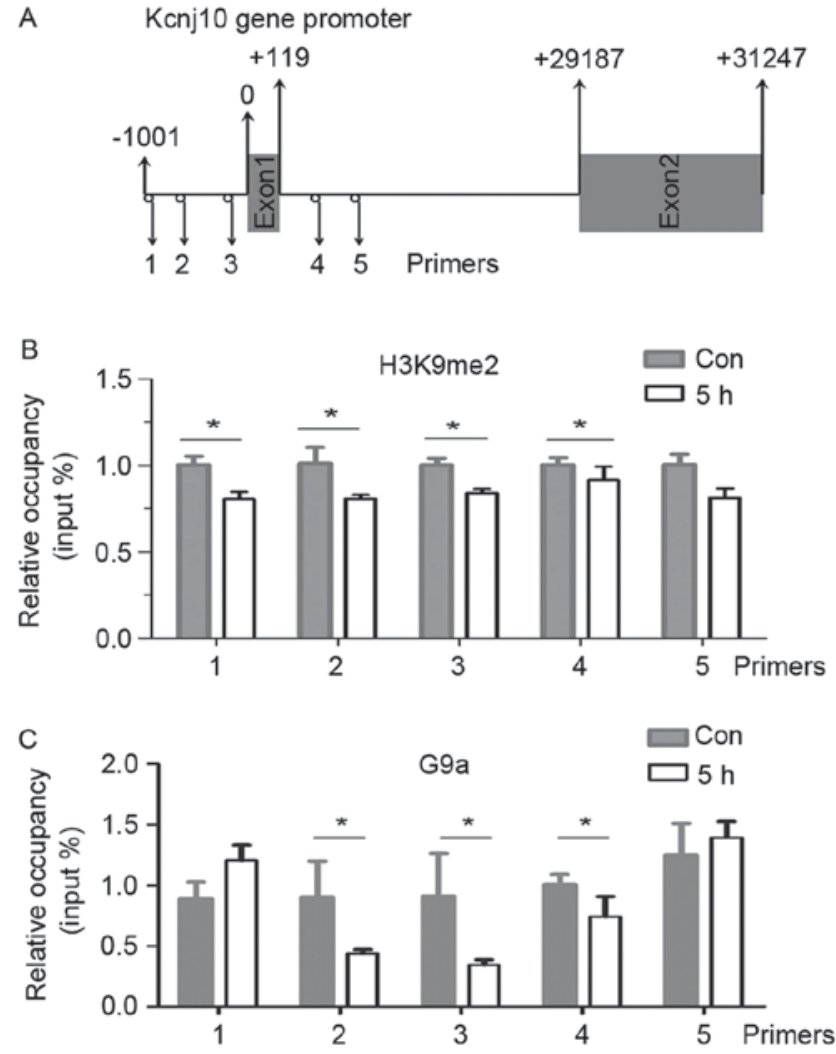

Figure 4. ChIP results demonstrating the relative abundance of $\mathrm{H} 3 \mathrm{~K} 9 \mathrm{me} 2$ and G9a in the promoter region of the Kcnj10 gene. (A) Visualized localization of reverse transcription-quantitative polymerase chain reaction primers in the promoter region of Kcnj10. (B) The relative occupancy of H3K9me2 and (C) G9a in the promoter region of Kcnj10. Genome DNA was used as an input. Five rats were used in each group $(n=5)$. Data are expressed as the mean \pm standard error. ${ }^{*} \mathrm{P}<0.05$ vs. the control group. ChIP, chromatin immune-precipitation; H3K9me2 dimethylation of histone 3 lysine 9; G9a, euchromatic histone-lysine N-methyltransferase 2; Kcnj10, ATP-sensitive inward rectifier potassium channel 10; Con, control.

and then returned to its original level after 1 day. A likely explanation for these observations is that $\mathrm{H} 3 \mathrm{~K} 9 \mathrm{me} 2$ and $\mathrm{G} 9 \mathrm{a}$ were sensitive to the stress caused by seizure, and responded to modulate the acute phase of epilepsy. Similar mechanisms have been previously reported in a study investigating seizures in cocaine addicts (15). In mouse nucleus accumbens, repeated cocaine administration reduces overall levels of $\mathrm{H} 3 \mathrm{~K} 9$ dimethylation catalysed by G9a, and the acute response may be mediated by the immediate FosB proto-oncogene (15).

An alternative mechanism of G9a and $\mathrm{H} 3 \mathrm{~K} 9 \mathrm{me} 2$ response to epileptic activity is through RE1 silencing transcription factor (REST). REST can respond to epileptic activity within $5 \mathrm{~h}$, and recruit co-repressors including HDACs, KDMs, Brg1 and G9a to become more abundant in dentate granule neurons (16). G9a alone cannot directly bind to DNA and needs to be recruited by a transcription factor, such as REST (17). In the present study, the expression of G9a was decreased, suggesting that there may be a REST-independent mechanism of G9a binding.

Potassium channels may be regulated by G9a and H3K9me2. In a neuropathic pain model, persistent pain stimulus can lead to a continuous upregulation of G9a and H3K9me2, and downregulation of some subtypes of potassium channels, such as Kcnq4, Kcnd2, Kcnq2 and Kcnq1 in dorsal root ganglion
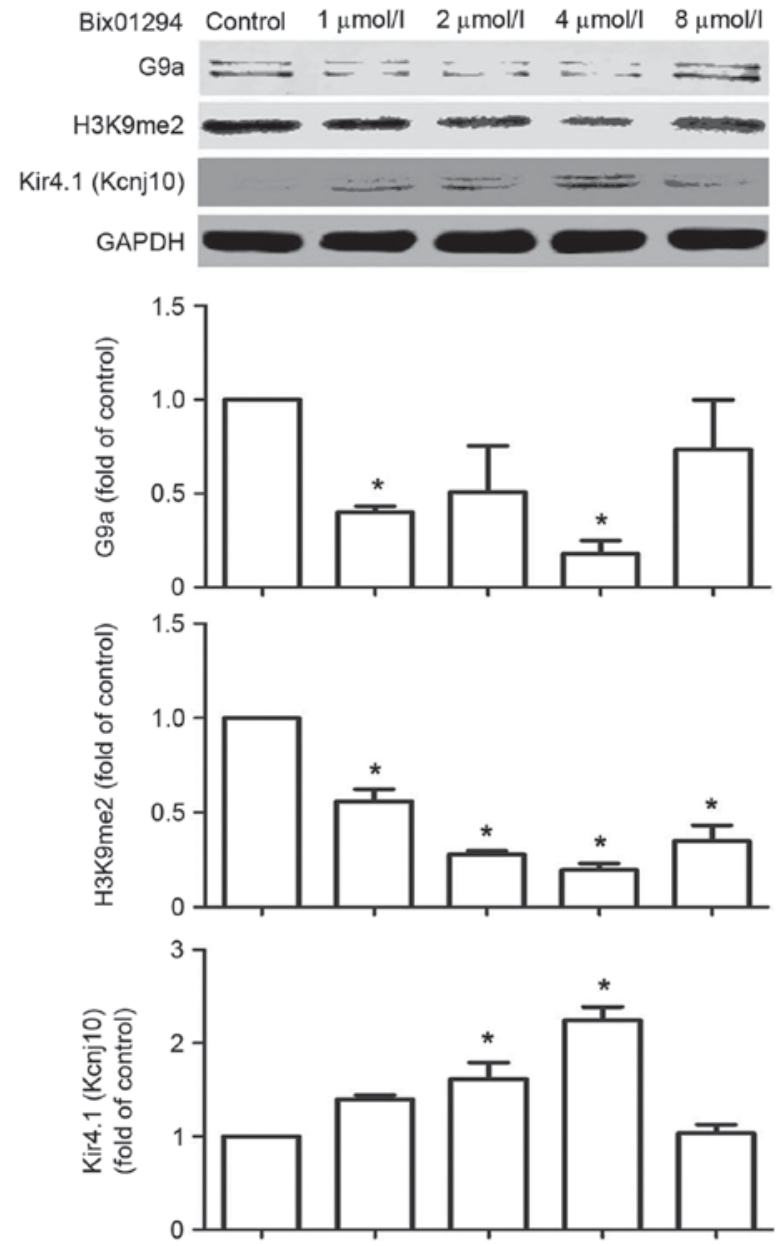

Figure 5. Western blotting of G9a, H3K9me2 and Kcnj10 following inhibition with Bix01294. C6 cells were stimulated with 1,2,4 or $8 \mu \mathrm{mol} / \mathrm{BBix} 01294$ for $5 \mathrm{~h}$. Data are expressed as the mean \pm standard error. ${ }^{*} \mathrm{P}<0.05$ vs. the control group. G9a, euchromatic histone-lysine N-methyltransferase 2; H3K9me2 dimethylation of histone 3 lysine 9; Kcnj10, ATP-sensitive inward rectifier potassium channel 10; Bix01294, 2-(Hexahydro-4-methyl-1H-1,4-diazepin-1-yl)-6,7-di-methoxy-N-(1-(phenylmethyl)-4-piperidinyl)-4-quinazolinamine tri-hydrochloride hydrate.

neurons (9). The screening performed in the present study demonstrated that Kcnj10 was upregulated while G9a and H3K9me2 were downregulated. ChIP assay demonstrated that, following a seizure, $\mathrm{H} 3 \mathrm{~K} 9 \mathrm{me} 2$ in the promoter region of Kcnj10 gene decreased causing its de-repression.

Kir4.1 encoded by Kcnj10, is a potassium channel expressed on the membrane of the astrocytes. Kir4.1 is an inwardly rectifying ATP-activated potassium channel that is mainly expressed in astrocytes, and serves a crucial role in the regulation of potassium ion homeostasis during neuronal activity (6). The phenotype of the Kir4.1-knockout mouse is characterized by severe epilepsy (18). Mutations in the human Kcnj10 gene have been reported in patients suffering from mesial temporal lobe epilepsy (19). As part of a feedback mechanism, Kir4.1 could respond to epileptic stress and become upregulated. These results suggest that astrocytes can respond to seizure-induced $\mathrm{K}^{+}$fluctuation.

The association between G9a and H3K9me2 downregulation, and the upregulation of the Kir4.1 potassium channel suggest that G9a and $\mathrm{H} 3 \mathrm{~K} 9 \mathrm{me} 2$ mediate epileptic seizure-induced changes in the Kcnj10 expression. ChIP 
results obtained in the present study confirmed that G9a and $\mathrm{H} 3 \mathrm{~K} 9 \mathrm{me} 2$ accumulation in the Kcnj10 promoter region decreased $5 \mathrm{~h}$ following epileptic activity. Inhibition of G9a and H3K9me2 with bix01294 in astrocyte tumour cell line promoted the expression of Kcnj10 in a dose dependent manner, which further confirmed the inhibitory regulation of G9a and H3K9me2 on Kcnj10 transcription.

The other upregulated gene was Kcnk10, which encodes for arachidonic acid sensitive TREK-2 $\mathrm{K}(+)$ potassium channel. The TREK channels are expressed highly in the human central nervous system, and can be activated by temperature, membrane stretch and internal acidosis (20). Due to its differences from Kir4.1, and unknown functions that need further investigation Trek2/Kcnk10 was not investigated further in the present study.

In conclusion, $\mathrm{H} 3 \mathrm{~K} 9 \mathrm{me} 2$ and $\mathrm{G} 9 \mathrm{a}$ are sensitive to epileptic seizure activity during the acute phase of epilepsy and can affect the transcriptional regulation of the Kcnj10 (Kir4.1) channel. Our findings may provide a potential epigenetic link between the regulation of potassium channel subtypes and epileptic development which may be helpful for understanding epilepsy.

\section{Acknowledgements}

The present study was funded by the National Natural Science Foundation of China (grant nos. 31460300 and 31260246); the Ningxia Medical University Foundation of China (grant nos. XY201406 and XY2016055); the National Basic Research Program of China (973 Program; grant no. 2012CB722408) and the 13.5 Major Scientific and Technological Projects of Ningxia Hui Autonomous Region (grant no. 2016BZ07).

\section{References}

1. Villa $\mathrm{C}$ and Combi R: Potassium channels and human epileptic phenotypes: An updated overview. Front Cell Neurosci 10: 81 , 2016.

2. Raimondo JV, Burman RJ, Katz AA and Akerman CJ: Ion dynamics during seizures. Front Cell Neurosci 9: 419, 2015.

3. Gorter JA, van Vliet EA, Aronica E, Breit T, Rauwerda H, Lopes da Silva FH and Wadman WJ: Potential new antiepileptogenic targets indicated by microarray analysis in a rat model for temporal lobe epilepsy. J Neurosci 26: 11083-11110, 2006.

4. Yang KC and Nerbonne JM: Mechanisms contributing to myocardial potassium channel diversity, regulation and remodeling. Trends Cardiovasc Med 26: 209-218, 2016.

5. Ryland KE, Hawkins AG, Weisenberger DJ, Punj V, Borinstein SC, Laird PW, Martens JR and Lawlor ER: Promoter methylation analysis reveals that KCNA5 ion channel silencing supports ewing sarcoma cell proliferation. Mol Cancer Res 14: 26-34, 2016.
6. Olsen ML and Sontheimer H: Functional implications for Kir4.1 channels in glial biology: From $\mathrm{K}^{+}$buffering to cell differentiation. J Neurochem 107: 589-601, 2008.

7. Nwaobi SE, Lin E, Peramsetty SR and Olsen ML: DNA methylation functions as a critical regulator of Kir4.1 expression during CNS development. Glia 62: 411-427, 2014.

8. Cadet JL, Brannock C, Krasnova IN, Jayanthi S, Ladenheim B, McCoy MT, Walther D, Godino A, Piroozina M and Lee RS: Genome-wide DNA hydroxymethylation identifies potassium channels in the nucleus accumbens as discriminators of methamphetamine addiction and abstinence. Mol Psychiatry 22: 1196-1204, 2017.

9. Laumet G, Garriga J, Chen SR, Zhang Y, Li DP, Smith TM, Dong Y, Jelinek J, Cesaroni M, Issa JP and Pan HL: G9a is essential for epigenetic silencing of $\mathrm{K}(+)$ channel genes in acute-to-chronic pain transition. Nat Neurosci 18: 1746-1755, 2015.

10. Fatima N, Cohen DC, Sukumar G, Sissung TM, Schooley JF Jr, Haigney MC, Claycomb WC, Cox RT, Dalgard CL, Bates SE and Flagg TP: Histone deacetylase inhibitors modulate KATP subunit transcription in HL-1 cardiomyocytes through effects on cholesterol homeostasis. Front Pharmacol 6: 168, 2015.

11. Bulk E, Ay AS, Hammadi M, Ouadid-Ahidouch H, Schelhaas S, Hascher A, Rohde C, Thoennissen NH, Wiewrodt R, Schmidt E, et al: Epigenetic dysregulation of $\mathrm{KCa} 3.1$ channels induces poor prognosis in lung cancer. Int J Cancer 137: 1306-1317, 2015.

12. Shankar SR, Bahirvani AG, Rao VK, Bharathy N, Ow JR and Taneja R: G9a, a multipotent regulator of gene expression. Epigenetics 8: 16-22, 2013.

13. Zhang L, Feng D, Tao H, DE X, Chang Q and Hu Q: Increased stathmin expression strengthens fear conditioning in epileptic rats. Biomed Rep 3: 28-32, 2015.

14. Livak KJ and Schmittgen TD: Analysis of relative gene expression data using real-time quantitative PCR and the 2(-Delta Delta C(T)) method. Methods 25: 402-408, 2001.

15. Maze I, Covington HE III, Dietz DM, LaPlant Q, Renthal W, Russo SJ, Mechanic M, Mouzon E, Neve RL, Haggarty SJ, et al: Essential role of the histone methyltransferase G9a in cocaine-induced plasticity. Science 327: 213-216, 2010.

16. Roopra A, Dingledine R and Hsieh J: Epigenetics and epilepsy. Epilepsia 53 (Suppl 9): S2-S10, 2012.

17. Hwang JY, Aromolaran KA and Zukin RS: Epigenetic mechanisms in stroke and epilepsy. Neuropsychopharmacology 38: 167-182, 2013.

18. Haj-Yasein NN, Jensen V, Vindedal GF, Gundersen GA, Klungland A, Ottersen OP, Hvalby $\mathrm{O}$ and Nagelhus EA: Evidence that compromised $\mathrm{K}^{+}$spatial buffering contributes to the epileptogenic effect of mutations in the human Kir4.1 gene (KCNJ10). Glia 59: 1635-1642, 2011.

19. Guo Y, Yan KP, Qu Q, Qu J, Chen ZG, Song T, Luo XY, Sun ZY, Bi CL and Liu JF: Common variants of KCNJ10 are associated with susceptibility and anti-epileptic drug resistance in Chinese genetic generalized epilepsies. PLoS One 10: e0124896, 2015.

20. Franks NP and Honoré E: The TREK K2P channels and their role in general anaesthesia and neuroprotection. Trends Pharmacol Sci 25: 601-608, 2004. 